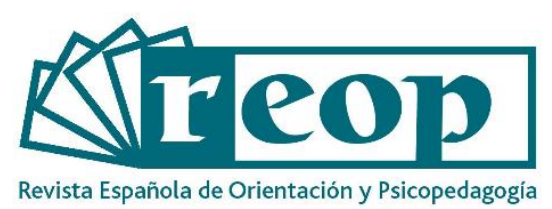

\title{
EFECTIVIDAD DE UN PROGRAMA PARA LA MEJORA DEL PROYECTO PROFESIONAL Y LA MARCA PERSONAL
}

\section{EFFECTIVITY OF A PROGRAM FOR THE IMPROVEMENT OF THE PROFESSIONAL PROJECT AND THE PERSONAL BRAND}

\author{
Fermín Carrillo González ${ }^{1}$ \\ Universidad de Zaragoza. Instituto Superior de Estudios Psicológicos. Barcelona, España
}

\section{RESUMEN}

La investigación propone el diseño y la aplicación de un programa de orientación profesional para mejorar el proyecto profesional y la marca personal, con el fin de aportar evidencias empíricas de su efectividad en la empleabilidad de un colectivo de psicólogos y educadores, estudiantes de postgrado, de una institución de educación superior. Se construyó un instrumento con escalas, unas ya existentes y otras elaboradas para la ocasión. Se realizó un estudio cuasiexperimental con diseño pretest-postest con un grupo experimental $(n=74)$ y otro control $(N=63)$. Los participantes fueron evaluados previamente al programa, y 6 meses tras la finalización de este. Como resultados se ha hallado que las escalas utilizadas tienen una adecuada estructura interna y fiabilidad. Los datos del análisis de modelos lineales generales de medidas repetidas (MLG), las pruebas t para muestras relacionadas y las d de Cohen dan lugar a resultados significativos: se han encontrado mejoras en el grupo experimental, tanto en el proyecto profesional y de marca personal, y en ciertas variables de empleabilidad. Así mismo, en el análisis del cambio tras realizar el programa, se ha hallado un modelo SEM en el que la asistencia al programa tiene un efecto estandarizado directo significativo en el desarrollo del proyecto $(B=.0,25)$ y en la satisfacción laboral $(B=.0,21)$, y de forma indirecta en el resto de las variables de empleabilidad. Se puede afirmar que, con esta muestra: el programa ha sido eficaz y efectivo, aunque hay que ser cautos, ya que los efectos podrían depender del perfil del participante.

\footnotetext{
${ }^{1}$ Correspondencia: Fermín Carrillo González. Correo-e: carrillofermin@gmail.com, fcarrillo@isep.es, web: $\underline{\text { www.isep.es }}$
} 
Palabras clave (negrita, arial 11): Orientación profesional, desarrollo profesional, programas de empleo, evaluación de programas, marketing.

\section{ABSTRACT}

The research proposes the design and application of a career guidance program to improve the professional project and personal branding, to provide empirical evidence of its effectiveness in the employability of a group of psychologists and educators, postgraduate students, from an institution of higher education. An instrument was built with scales, some already existing and others made for the occasion. A quasi-experimental study was carried out with a pretest-posttest design with an experimental group $(n=74)$ and another control $(N=63)$. The participants were evaluated before the program, and 6 months after the end of the program. As results it has been found that the scales used have adequate internal structure and reliability. The data of the analysis of general linear models of repeated measures (MLG), the t-tests for related samples and d Cohen give rise to significant results: improvements have been found in the experimental group, both in the professional and personal brand project, and in certain employability variables. Likewise, in the analysis of the change after completing the program, an SEM model has been found in which the assistance to the program has a significant direct standardized effect on the development of the project $(B=.0 .25)$ and on job satisfaction $(B=.0 .21)$, and indirectly in the rest of employability variables. It can be affirmed that, with this sample: the program has been effective, although it is necessary to be cautious, since the effects could depend on the profile of the participant.

Key Words: Career guidance, professional development, employment programs, program evaluation, marketing.

\section{Cómo citar este artículo:}

Carrillo González, F. (2021). Efectividad de un programa para la mejora del proyecto profesional y la marca personal. Revista Española de Orientación y Psicopedagogía, 32(2), 27-46. https://doi.org/10.5944/reop.vol.32.num.2.2021.31277

\section{Introducción}

Hay diversidad de estudios que pretenden identificar las variables y competencias que inciden en los procesos de inserción laboral y en la empleabilidad. Entre ellas, factores internos a los individuos para encontrar y mantener un trabajo (Song y Ling, 2013, Scharr y Bartlet, 2014; Hodzic et al., 2015; Bell, 2016; Sun et al., 2016; Nisha y Rajasekaran, 2018, entre muchos otros) y las variables externas, relacionadas con el macrocontexto (Figuera, 1996) y los factores de ocupabilidad (Romero et al., 2004) relativos a elementos económicos, políticos, sociales, demográficos, etc. sobre los que el individuo no tiene control.

Con esta investigación queremos saber los efectos de desarrollar el proyecto profesional y la marca personal en variables relacionadas con la empleabilidad, mediante el desarrollo y la evaluación de un programa de orientación profesional. 


\section{Enfoque propuesto de orientación profesional}

La tasa de desempleo en España está aumentando por la crisis sanitaria del COVID-19 y se sitúa en el 15,3\% (INE, 2020). Existen estudios en los que se ha encontrado una alta insatisfacción laboral entre los trabajadores, que llega al 25\% (Randstad, 2018), o en los que se manifiestan entre los más jóvenes expectativas muy bajas de empleo en las carreras cursadas (Natalya y María, 2013). Se acepta que el modelo clásico de un trabajo fijo para toda la vida ha llegado a su fin, y es necesario adaptarse a las continuas crisis económicas y a las transiciones del mercado laboral (Sobrado y Ceinós, 2017), mediante la adquisición de nuevas competencias profesionales exigidas por los constantes cambios tecnológicos, sociales y económicos (Echeverría, 2008).

La orientación profesional ha querido abordar esta problemática, en un contexto de globalización, transformación social y de nuevas formas y métodos de trabajo, y ha concluido que su función esencial es la de ayudar a los individuos en la construcción de su proyecto profesional y de su gestión de la carrera, en este mundo cambiante (Álvarez y Sánchez, 2017). Los enfoques de intervención deben evolucionar (Sobrado y Cortés, 2009) hacia modelos más holísticos (Krumboltz et al., 2013), para la adquisición de competencias para el desarrollo de la carrera (Nickson et al., 2012), a lo largo de toda la vida (McCarthy, 2016), para todos los individuos (Álvarez y Sánchez, 2017) y buscando la continua autogestión y la autoorientación responsable (Sobrado y Ceinós, 2017).

Existen modelos de intervención que tienen como fin ayudar para lograr estos objetivos. Por ejemplo, el modelo de programas para mejorar el proyecto profesional y de vida (Romero, 2004) pretende que los participantes aprendan a anticiparse al mercado, construyan su proyecto laboral y vital, y actúen en consecuencia. O los modelos de gestión personal de la carrera, que procuran que los individuos exploren sus posibilidades en el contexto, establezcan metas y estrategias y evalúen su implementación (Greenhaus et al., 2010; Savickas, 2012; Pinto et al., 2016).

En este sentido, estamos totalmente de acuerdo con Rodríguez et al. (2020), que necesitamos nuevos enfoques en la orientación educativa y vocacional para ayudar a las personas a diseñar su propio proyecto de vida y profesional, en un mundo digital, que integren elementos de la psicología positiva y que pretendan evaluar y mejorar el bienestar.

La comprensión de la inserción profesional ha generado teorías clásicas como las del capital humano (énfasis en la educación), las credencialistas (importancia de las titulaciones) y las de la correspondencia (basadas en la incidencia del origen social y cultural); pero han evolucionado a otras que procuran encontrar las explicaciones en las diferencias en los individuos: la autoestima, la experiencia previa, el apoyo social y la identidad (Lazarus y Folkman, 1986); el sentimiento de autoeficacia (Bandura, 1977); la percepción de la propia competencia (Van del Heijde y Van der Haijden, 2006); la actitud hacia el trabajo y su adaptación al mismo (Fugate y Kinicki, 2008; Bermúdez López et al., 2020) y la empleabilidad percibida (Vanhercke et al., 2014). Justamente esta última referencia dio mucha importancia a la propia competencia percibida, a la actitud hacia el mercado de trabajo y a la empleabilidad percibida (sentimiento de capacidad para conseguir empleo). Las dos primeras variables serían de entrada, que afectarían a la empleabilidad percibida, y esta afectaría a la activación de los recursos de la persona para mejorar su empleabilidad. Todo ello, mediatizado por los efectos de la situación y el entorno. Sin duda, la empleabilidad percibida es una variable importante por estar relacionada con las expectativas de logro en la inserción (Izquierdo y Farias, 2018). 


\section{Empleabilidad e importancia de la marca personal}

La empleabilidad ha sido estudiada desde diferentes disciplinas (psicología, educación, sociología, economía, política, ...) y perspectivas (Suárez, 2014) y, por tanto, hay diversas acepciones. En nuestra investigación se comprende desde una triple perspectiva: como la capacidad para conseguir o mantener un trabajo y saberse desenvolver en el mercado laboral (OEEU, 2015); como la competencia de la persona para satisfacer sus necesidades de desarrollo profesional (Sáez y Torres, 2007); y como la percepción que uno tiene de recibir oportunidades laborales (Gamboa et al., 2007).

Consideramos clave para entender el nuevo enfoque propuesto que hay un $75-85 \%$ de ofertas laborales en el mercado de trabajo que son ocultas y no son difundidas por canales públicos de información (Lee Hecht Harrison, 2017). Estas son inaccesibles mediante los procesos de búsqueda de empleo clásicos (como, por ejemplo, webs de empleo o bolsas de trabajo). Sólo se pueden conocer mediante canales informales, donde las redes sociales y el networking adquieren mucha importancia. El objetivo de los candidatos para recibir dichas oportunidades laborales clandestinas es estar en la mente de quienes ofrecen dichas oportunidades (Pérez Ortega, 2015). Para este sistema, seguramente sea más provechoso desarrollar estrategias relacionadas con el posicionamiento, la marca personal, el desarrollo de competencias digitales (Juárez y Marqués, 2019) y el desarrollo de la red de contactos virtual y offline. Este último canal es el más importante para la inserción según los estudios (Fernández-Salinero y García Álvarez, 2020), al permitir acceder a información privilegiada y a recursos inaccesibles, de otro modo (Batistic y Tymon, 2017).

La marca personal es el resultado de un proceso en el que adquiere importancia la visibilidad (Peters, 1997). La promoción profesional permite conocer, al que ofrece oportunidades de trabajo, el tipo de ayuda que puede recibir; y obtiene pruebas de la calidad, los conocimientos y habilidades del que se hace visible (Pérez Ortega, 2015). La reputación, el cómo se es percibido por los demás, depende justamente de dicho proceso (Arqués, 2019). La marca personal permite saber qué es lo que se ofrece (Gander, 2014) y se reduce la incertidumbre de lo desconocido permitiendo una mejor toma de decisiones, tanto por un mejor ajuste de las expectativas (Pérez Ortega, 2015), como por la emoción que genera todo este proceso (Deckres y Lacy, 2014) en el empleador o cliente. En el branding personal se pueden establecer tres fases: la autoevaluación profesional, la elección de posicionamiento y la de comunicación (Peters, 1997). Más recientemente, Pérez Ortega (2015) con su modelo ADN, habla de Análisis de atributos, Diferenciación, y Notoriedad. En cualquiera de los dos modelos, se hace patente la importancia del autoconocimiento, la determinación del modo como uno quiere ser reconocido y la promoción.

Existen críticas hacia el enfoque de la marca personal como factor de empleabilidad. Este ha sido considerado como muy comercial y vinculado con prácticas poco éticas (Cederberg, 2017); propio de divulgadores que se dirigen a un público de manera persuasiva y sin considerar sus perfiles ni la realidad de cada uno de ellos (Gershon, 2017). Conley (2009) afirma que la marca personal es un constructo desorganizado y demasiado optimista; y Zarkada (2012) que las evidencias científicas de sus efectos son muy débiles.

\section{Programa para el desarrollo del proyecto profesional y la marca personal}

El programa propuesto para la investigación tiene como objetivo que los participantes elaboren e implementen su proyecto profesional, además de su marca personal, con el objetivo de aumentar su empleabilidad. Los contenidos trabajados incluyen una introducción a la realidad sociolaboral, los factores de la empleabilidad, el concepto de marca personal y lo que contempla, y la explicación de cada una de las fases de desarrollo del proyecto profesional: establecimiento de la misión 
profesional, la visión, análisis del entorno, diagnóstico DAFO, establecimiento de objetivos, definición de estrategias y elaboración del plan de acción.

El programa constó de 11 horas presenciales realizadas en dos sesiones de 5 y 6 horas, donde los participantes realizaron, con ayuda del profesor, su proyecto profesional y de marca personal. En una segunda fase del programa, que duró unos 6 meses, el orientador estuvo disponible para cualquier consulta de los estudiantes respecto a su proyecto, y su implementación.

El programa y la investigación se puede considerar que sigue la misma línea de trabajo que la de Climent-Rodríguez y Navarro-Abal (2016) en cuanto a programas de inserción laboral y empleabilidad, que buscan que los participantes desarrollen sus marcas personales y aprendan a diferenciarse en un mercado cada vez más competitivo.

\section{Método}

Esta investigación pretende evaluar la eficacia y efectividad de un programa para mejorar el proyecto profesional, la marca personal y la empleabilidad en un colectivo de profesionales de la psicología y la educación de un instituto de educación superior, de postgrado.

La hipótesis principal es que el programa será eficaz y los participantes del grupo experimental desarrollarán más su proyecto profesional y de branding personal, que los del grupo control. Y que el programa será efectivo, por lo que los participantes mejorarán indicadores de empleabilidad de forma más significativa que los del grupo control.

\section{Metodología}

Para alcanzar el objetivo se ha partido de las necesidades sociales y de empleo de la muestra de estudiantes que habían cursado o estaban realizando un programa de máster, así como del contexto de la institución, para diseñar un programa de orientación profesional. Tras su diseño, se realizó la evaluación de entrada o del diseño mediante una comparación de su estructura con los programas de orientación profesional basados en los proyectos profesionales y de vida (Romero et. al. 2004), y en los programas basados en la gestión personal de la carrera (Greenhaus et al., 2010). Adicionalmente, once expertos evaluaron el programa mediante una adaptación de la escala de evaluación de programas de Maquilón (2005).

Para la evaluación de la aplicación del programa, o evaluación del proceso, se realizaron dos cursos pilotos para reajustar parámetros de su implementación. Con la información obtenida, finalmente se desarrolló el programa con los participantes del grupo experimental, y se evaluó su aplicación. No hubo asignación aleatoria de los sujetos a cada uno de los grupos.

Se construyó un instrumento con escalas, unas ya existentes y otras elaboradas para la ocasión, tomando como referencia el marco teórico. Para evaluar los resultados del programa, y especialmente la eficacia y la efectividad del mismo, se realizó un estudio cuasiexperimental con un diseño pretest-postest (previamente al desarrollo del programa y seis meses más tarde de la aplicación del mismo) con un grupo experimental y otro grupo control, siendo la variable independiente el asistir a este programa en el que el alumno desarrolla su proyecto profesional y la marca personal. Las variables dependientes son indicadores de empleabilidad: oportunidades 
laborales recibidas, satisfacción laboral, reputación percibida, número de clientes en los últimos 6 meses, situación laboral y vinculación con su proyecto profesional y grado de mejora de la situación laboral conforme a su proyecto profesional. Se han integrado factores modeladores de nuestro modelo teórico, como son la percepción general de competencia profesional, la actitud hacia el mundo del trabajo, la empleabilidad percibida, el desarrollo del proyecto profesional y la marca personal, y las actividades de promoción del participante. Aparte, variables sociobiográficas.

\section{Participantes}

El grupo experimental (GE) contó con 74 participantes y el grupo experimental (GC), con 63. El GE fue dividido, para facilitar el desarrollo del programa, en 3 subgrupos. Las características básicas de todos los grupos se resumen en la tabla 1.

\section{Tabla 1}

Descripción de los diferentes grupos de la investigación

\begin{tabular}{|c|c|c|}
\hline Sujetos & $\begin{array}{l}\text { GE } \\
74\end{array}$ & $\begin{array}{l}\mathrm{GC} \\
63\end{array}$ \\
\hline Mujeres (\%) & $89,2 \%$ & $93,7 \%$ \\
\hline Hombres (\%) & $10,8 \%$ & $6,3 \%$ \\
\hline Nacionalidad Española (\%) & $74,3 \%$ & $87,3 \%$ \\
\hline Otras nacionalidades (\%) & $25,7 \%$ & $12,7 \%$ \\
\hline Residencia fuera de España & $6,8 \%$ & $9,5 \%$ \\
\hline Profesionales de la Psicología (\%) & $62,2 \%$ & $69,8 \%$ \\
\hline Profesionales de la educación y salud (\%) & $21,5 \%$ & $23,9 \%$ \\
\hline Otras profesiones (\%) & $14,9 \%$ & $6,3 \%$ \\
\hline Sin acabar estudios (\%) & $1,4 \%$ & $0 \%$ \\
\hline Con Máster finalizado (\%) & $56,8 \%$ & $60,3 \%$ \\
\hline Media de edad (años) & 37,7 & 32,2 \\
\hline Desviación típica de la edad & 11,3 & 8,5 \\
\hline Edad mínima (años) & 22 & 22 \\
\hline Edad máxima (años) & 62 & 55 \\
\hline Media de años en profesión deseada & 6,5 & 2,7 \\
\hline Desviación típica de años en profesión deseada & 8,8 & 3,9 \\
\hline Hijos menores de 12 años (\%) & $13,5 \%$ & $23,8 \%$ \\
\hline Discapacidad (\%) & $1,4 \%$ & $0 \%$ \\
\hline Problemas de salud (\%) & $0 \%$ & $1,6 \%$ \\
\hline
\end{tabular}

\section{Procedimientos}

Los elementos de la evaluación que se contemplaron fueron: la evaluación de la eficacia, para valorar los aprendizajes; en este caso, que mejoren su proyecto profesional y de marca personal. $Y$ la evaluación de la efectividad: para determinar el grado de transferencia de los aprendizajes a su contexto real; en otras palabras, si el programa tiene efectos en las variables del estudio, especialmente en la empleabilidad.

Se utilizó un cuestionario que los participantes del grupo control y experimental contestaron dos veces: previamente al programa, y al menos seis meses más tarde. El objeto era evaluar el grado de desarrollo del proyecto profesional y de la marca personal; y las diversas variables mediadoras 
y de empleabilidad. Se analizaron las posibles diferencias significativas entre el pretest y postest en las escalas, las interacciones significativas del modelo lineal general (MLG) de medidas repetidas, y el análisis de la D de Cohen.

Así mismo, se realizó un análisis de ecuaciones estructurales para encontrar un modelo explicativo de los efectos significativos de la variable independiente (la asistencia al programa de los participantes) en el resto de las variables de la investigación.

\section{Instrumentos}

Las variables del cuestionario, que se han pretendido evaluar en el pretest y en el postest, se representan en el Figura 1. Se indican las escalas utilizadas.

\section{Figura 1}

Variables del estudio y sus relaciones teóricas e instrumentos utilizados para su medición

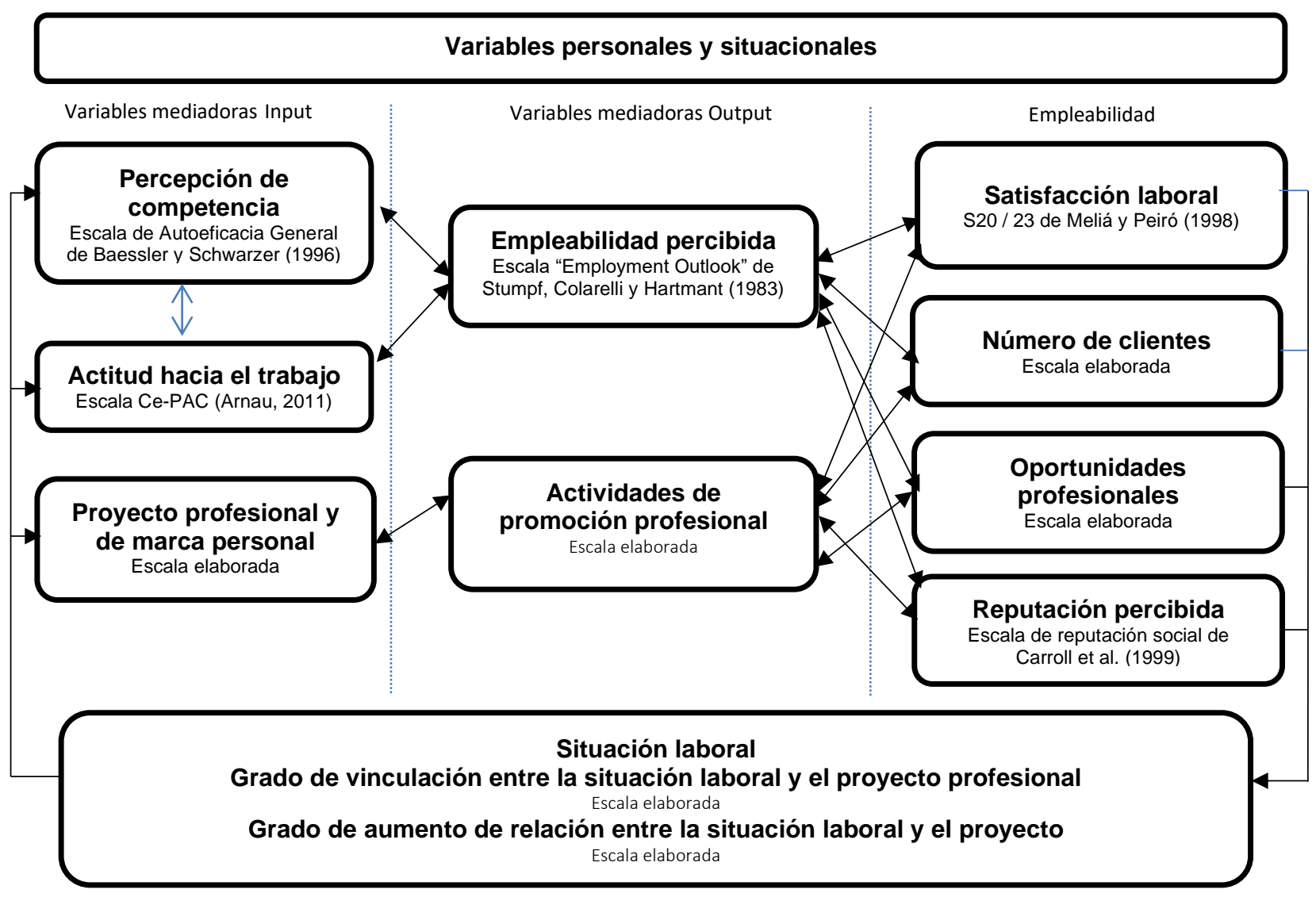

Fuente: Elaboración propia

A continuación, una breve descripción:

Variables Mediadoras ínputs

Estas variables hacen referencia a los factores de entrada que Vanhercke et al. (2014) indicaban que precedían a la empleabilidad percibida: 


\section{- Percepción de competencia General}

Relativa a los pensamientos y creencias que un individuo tiene sobre sus habilidades y que tienen una influencia en su comportamiento. La escala utilizada es una adaptación para la población española de la Escala de Autoeficacia General de Baessler y Schwarzer (1996) realizada por Sanjuan et al. (2000).

- Actitud hacia el trabajo

Es la disposición de la persona a adaptarse al mundo del trabajo, evaluada a partir de la adaptación española de la escala que Vanhercke et al. (2014) sugirieron: el Ce-PAC (Arnau, 2011).

\section{- Proyecto Profesional y Branding Personal}

Si bien existen ciertos cuestionarios para evaluar la planificación de la carrera (Rodríguez Moreno, 2008) no existe ninguno que integre y comprenda los elementos que conforman cada una de las fases de un proyecto profesional y de creación de la marca personal. Por ello, se hizo necesario construir una escala para evaluar su grado de desarrollo

En la evaluación del cuestionario piloto se evaluó la escala construida y finalmente se mejoró hasta obtener una escala fiable y validada.

\section{Variables mediadoras output}

Las variables output están condicionadas por las variables input: la percepción de uno mismo en cuanto a las competencias y las actitudes hacia el trabajo.

- Empleabilidad percibida

Se refiere a las creencias de las personas para alcanzar sus metas profesionales (Vanhercke et al., 2014). Se utilizaron dos ítems de la Escala "Employment Outlook" de Stumpf et al. (1983), que tiene una escala consistencia interna de $\alpha=, 76$

- Actividades de promoción

Son aquellas acciones que una persona realiza conforme al proyecto profesional con el fin de dar a conocer lo que uno conoce o sabe hacer. Para ello se construyó una escala para medir y conocer el número de acciones de promoción en diferentes canales (ofrecimiento para asistir a eventos, realizar publicaciones impresas, dictar ponencias, participar en medios de comunicación, o tener colaboraciones con instituciones y empresas). Se redactaron, analizaron y seleccionaron los ítems definitivos aplicados sobre una muestra piloto, tras calcular su fiabilidad y analizar su estructura factorial.

\section{Variables de empleabilidad}

\section{- Satisfacción laboral}

Puede ser considerada como una evidencia de la empleabilidad en cuanto a que el individuo valora su situación actual de trabajo como favorable. Para evaluarla se tomaron 4 ítems del cuestionario S20 / 23 de Meliá y Peiró (1998) que tienen un carácter genérico y que permite recabar información sobre su satisfacción general laboral y sus ingresos.

- Recepción de oportunidades

Se construyó una escala en donde se le preguntaba al participante cuantas veces había recibido en los últimos 6 meses solicitudes para dictar conferencias, participar en algún congreso, escribir un 
libro o un artículo, colaborar en investigaciones, asumir un cargo, etc. Difiere de la escala actividades de promoción en que en esta el sujeto se ofrece voluntariamente a los demás, mientras que en la recepción de oportunidades el profesional es requerido por clientes o empleadores, que le ofrecen colaboración.

- Número de clientes

Tiene que ver con el número de personas/empresas/instituciones que te reclaman, te pagan o te eligen para ocupar un puesto de trabajo o actividad profesional.

- Reputación percibida

Se integraron los 4 ítems de la escala de reputación social de Carroll et al. (1999) que, si bien está construida para adolescentes, nos puede servir para evaluar el grado de percepción que tiene cada sujeto sobre su popularidad y el grado de marca personal que pudiera tener el participante.

- Situación laboral y vinculación con su proyecto profesional

Esta escala, construida para la ocasión, desea evaluar en el participante el grado en el que en los últimos 6 meses su realidad laboral está conforme a su proyecto profesional.

\section{- Grado de mejora de la situación laboral conforme a su proyecto profesional}

Es el grado en el que en los últimos 6 meses se ha mejorado la situación profesional, respecto a su proyecto profesional.

Para realizar la validación de las escalas se contó con 357 participantes que respondieron el cuestionario en un estudio previo. Se realizó el análisis factorial exploratorio de las nuevas escalas, encontrando que las escalas tienen una estructura adecuada interna. Así mismo, demostraron una fiabilidad interna aceptable, con un Alfa de Cronbach superior a 0,80 y una estabilidad temporal entre los datos del pretest y del postest, con un índice de correlación positivo y significativo estadísticamente con un $5 \%$ de margen de error.

\section{Resultados}

En primer lugar, se comprobó que todos los subgrupos del grupo experimental (GE) cumplieran con la distribución normal aplicando la prueba de Kolmogorov-Smirnov. También se realizó la comparativa de las medias del pretest para las diferentes escalas entre los 3 subgrupos del GE, y no se encontraron diferencias significativas. Se realizó una tabla de contingencia y se calculó el Chicuadrado y los 3 subgrupos no difirieron en las variables cualitativas (sexo, situación laboral actual, estudios, nacionalidad, país de residencia, postgrado acabado, hijos menores de 12 años, etc.). Por tanto, se consideraron homogéneos y se integraron en el mismo GE.

Para averiguar el grado de homogeneidad entre el GE y el GC se procedió a realizar el mismo análisis mediante la prueba t de muestras independientes y se concluyó que no diferían en los datos de las escalas, aunque sí en la edad y en los años que llevaban trabajando. El grupo GE tenía más edad $(\bar{X}=37,68)$, se graduó hace más años $(\bar{X}=10,79)$, llevaba más tiempo trabajando $(\bar{X}=14,11)$ y ejerciendo la profesión que deseaban realizar $(\bar{X}=6,50)$. También es de destacar que, aunque sin diferencias significativas en el pretest, el GC tenía puntuaciones mayores en ciertas variables de empleabildiad. Se podría considerar que asistieron al programa aquellas personas que comprendían más su necesidad y percibían que les iba a ser más útil. 
Así mismo, tras analizar la tabla de contingencias, se pudo afirmar que son dos grupos sin diferencias significativas en cuanto a las variables: sexo, situación laboral, estudios, nacionalidad, país de residencia, postgrado finalizado e hijos menores de 12 años. Por tanto, se puede concluir diciendo que ambos grupos tienen cierto grado de homogeneidad desde el punto de vista estadístico.

Mediante la utilización de la prueba T para muestras relacionadas se obtuvo en el GE una correlación positiva y significativa de todas las escalas del pretest con el postest, lo que es una evidencia de la estabilidad de las puntuaciones. Asimismo, se halló una mejora significativa, con un $\alpha=0,05$ de margen de error, entre los datos del pretest y del postest, en todas las escalas, excepto en la Escala de Sentimiento de Competencia y Reputación Percibida. Ello indica que los participantes del grupo experimental mejoraron en la mayoría de las variables de la investigación, probablemente debido al programa.

Los resultados de aplicar la prueba T para muestras relacionadas con el GC, muestran que también existe una correlación significativa entre el pretest y el postest. Pero en la significatividad de las diferencias entre las medias, no son mejores las puntuaciones del postest, excepto en la escala de oportunidades recibidas. Con estos datos, podríamos afirmar que no hay diferencias significativas entre los datos previos al desarrollo del programa y posteriores al mismo. Pero sí que hay diferencias en el GE, por lo que podrían existir evidencias de una influencia positiva del programa en la mejora de la mayoría de los indicadores propuestos en el estudio.

A continuación, en las tablas 2 y 3 , se exponen los resultados sintéticos de las medias, desviación típica, d de Cohen, y del análisis del Modelo Lineal General de los datos del GE y el GC. A partir de ellos, podemos afirmar que no se encontraron diferencias significativas en las variables percepción de competencia, actitud hacia el cambio, reputación percibida y escala de clientes, entre el GC y el GE. La d de Cohen de la variable empleabilidad percibida del GC es de 0,08, mientras que la del GE es de 0,38. Aparentemente la magnitud del efecto en el grupo experimental es significativamente mayor. No obstante, la diferencia no alcanza la significatividad estadística, de acuerdo a la interacción en el MLG, de "asistencia al programa". Sí que hay diferencias significativas entre la totalidad de participantes que han asistido al programa y en el postest tienen mayor empleabilidad percibida. Sucede lo mismo con "actividades de promoción", "oportunidades recibidas", "vinculación entre el proyecto y la realidad profesional" y "grado de mejora de la situación profesional con respecto al proyecto". En todos los casos los efectos del grupo experimental son superiores, hay una diferencia significativa de todos los participantes entre el postest y el pretest, pero no es relevante directamente el haber asistido al programa.

Por último, hay dos variables que el haber asistido al programa sí que afecta significativamente para tener mejores puntuaciones en el postest: la escala de desarrollo del proyecto profesional y la marca personal, y la escala de satisfacción profesional. Este dato se corrobora con el estadístico significativo del MLG de mejora entre el postest y el pretest. Y, especialmente, con la d de Cohen, que en el GE es de 0,81 en el primer caso, y 0,52 en el segundo. Son dos efectos de bastante magnitud, respecto al 0,21 y -0.01 del GC.

Tras este análisis, podemos afirmar sin temor a equivocarnos que el programa ha sido eficaz, desarrollando el proyecto profesional y de marca personal en el GE, con un $5 \%$ de margen de error, y ha afectado directamente a la satisfacción laboral.

En el gráfico 1 se muestran los efectos del programa en las variables en las que este ha interactuado significativamente. 


\section{Tabla 2}

Análisis de los efectos del programa en el GE respecto al GC

\begin{tabular}{|c|c|c|c|c|c|c|c|c|c|}
\hline & \multirow{3}{*}{$\begin{array}{l}\text { Tipo } \\
\text { de } \\
\text { grupo }\end{array}$} & \multirow{3}{*}{$\mathrm{N}$} & \multirow{3}{*}{ Media } & \multirow{3}{*}{ SD } & \multirow{3}{*}{$\begin{array}{l}\mathrm{d} d \mathrm{de} \\
\text { Cohe } \\
\mathrm{n}\end{array}$} & \multicolumn{4}{|c|}{ MODELO LINEAL GENERAL } \\
\hline & & & & & & \multicolumn{2}{|c|}{$\begin{array}{c}\text { Diferencias } \\
\text { Significativas } \\
\text { entre el } \\
\text { Postest y el } \\
\text { Pretest }\end{array}$} & \multicolumn{2}{|c|}{$\begin{array}{l}\text { Interacción } \\
\text { Asistencia al } \\
\text { Programa }\end{array}$} \\
\hline & & & & & & & SIG & $\mathrm{F}$ & SIG \\
\hline $\begin{array}{l}\text { Escala sentimiento } \\
\text { competencia }\end{array}$ & & & 79,60 & 10,048 & & \multirow{4}{*}{,025 } & \multirow{4}{*}{,875 } & \multirow{4}{*}{,162 } & \multirow{4}{*}{,688 } \\
\hline $\begin{array}{l}\text { Escala sentimiento } \\
\text { competencia post }\end{array}$ & GC & 63 & 79,40 & 11,632 & $-0,02$ & & & & \\
\hline $\begin{array}{l}\text { Escala sentimiento } \\
\text { competencia }\end{array}$ & \multirow{2}{*}{ GE } & \multirow{2}{*}{74} & 79,91 & 12,006 & \multirow{2}{*}{0,04} & & & & \\
\hline $\begin{array}{l}\text { Escala sentimiento } \\
\text { competencia post }\end{array}$ & & & 80,38 & 12,139 & & & & & \\
\hline Escala actitud hacia el cambio & & & 18,48 & 4,543 & & \multirow{4}{*}{1,661} & \multirow{4}{*}{ 200 } & \multirow{4}{*}{2,409} & \multirow{4}{*}{,123 } \\
\hline $\begin{array}{l}\text { Escala actitud hacia el cambio } \\
\text { post }\end{array}$ & GC & 63 & 18,38 & 4,531 & $-0,02$ & & & & \\
\hline Escala actitud hacia el cambio & \multirow[b]{2}{*}{ GE } & \multirow[b]{2}{*}{74} & 17,50 & 4,298 & \multirow[b]{2}{*}{0,24} & & & & \\
\hline $\begin{array}{l}\text { Escala actitud hacia el cambio } \\
\text { post }\end{array}$ & & & 18,53 & 4,145 & & & & & \\
\hline Escala empleabilidad percibida & & & 5,48 & 1,950 & & \multirow{4}{*}{5,772} & \multirow{4}{*}{,018 } & \multirow{4}{*}{2,646} & \multirow{4}{*}{ 106 } \\
\hline $\begin{array}{l}\text { Escala empleabilidad percibida } \\
\text { post }\end{array}$ & GC & 63 & 5,63 & 2,288 & 0,08 & & & & \\
\hline Escala empleabilidad percibida & \multirow[b]{2}{*}{ GE } & \multirow[b]{2}{*}{74} & 5,39 & 2,157 & \multirow[b]{2}{*}{0,38} & & & & \\
\hline $\begin{array}{l}\text { Escala empleabilidad percibida } \\
\text { post }\end{array}$ & & & 6,22 & 2,277 & & & & & \\
\hline Escala proyecto profesional & & & 18,03 & 5,883 & & \multirow{4}{*}{27,117} & & & \\
\hline $\begin{array}{l}\text { Escala proyecto profesional } \\
\text { post }\end{array}$ & GC & 63 & 19,24 & 5,312 & 0,21 & & (200 & $0 G 6 ?$ & ת० \\
\hline Escala proyecto profesional & & & 16,30 & 5,903 & & & , & 9,602 & , \\
\hline $\begin{array}{l}\text { Escala proyecto profesional } \\
\text { post }\end{array}$ & GE & 74 & 21,08 & 6,583 & 0,81 & & & & \\
\hline Escala actividades de & & & 7,79 & 6,008 & & & & & \\
\hline $\begin{array}{l}\text { promoción } \\
\text { Escala actividades de } \\
\text { promoción post }\end{array}$ & GC & 63 & 8,70 & 6,722 & 0,15 & & & & \\
\hline Escala actividades de & & & 6,81 & 6,130 & & 8,735 & 004 & 0,703 & 403 \\
\hline $\begin{array}{l}\text { promocion } \\
\text { Escala actividades de } \\
\text { promoción post }\end{array}$ & GE & 74 & 8,43 & 6,648 & 0,26 & & & & \\
\hline Escala reputación percibida & & & 9,89 & 2,984 & & & & & \\
\hline $\begin{array}{l}\text { Escala reputación percibida } \\
\text { post }\end{array}$ & GC & 63 & 10,19 & 3,141 & 0,10 & 010 & 207 & & 701 \\
\hline Escala reputación percibida & & & 9,82 & 3,485 & & , 819 & , &, 119 & , \\
\hline $\begin{array}{l}\text { Escala reputación percibida } \\
\text { post }\end{array}$ & GE & 74 & 9,96 & 3,450 & 0,04 & & & & \\
\hline
\end{tabular}

Fuente: Elaboración propia 


\section{Tabla 3}

Análisis de los efectos del programa en el GE respecto al GC (Continuación)

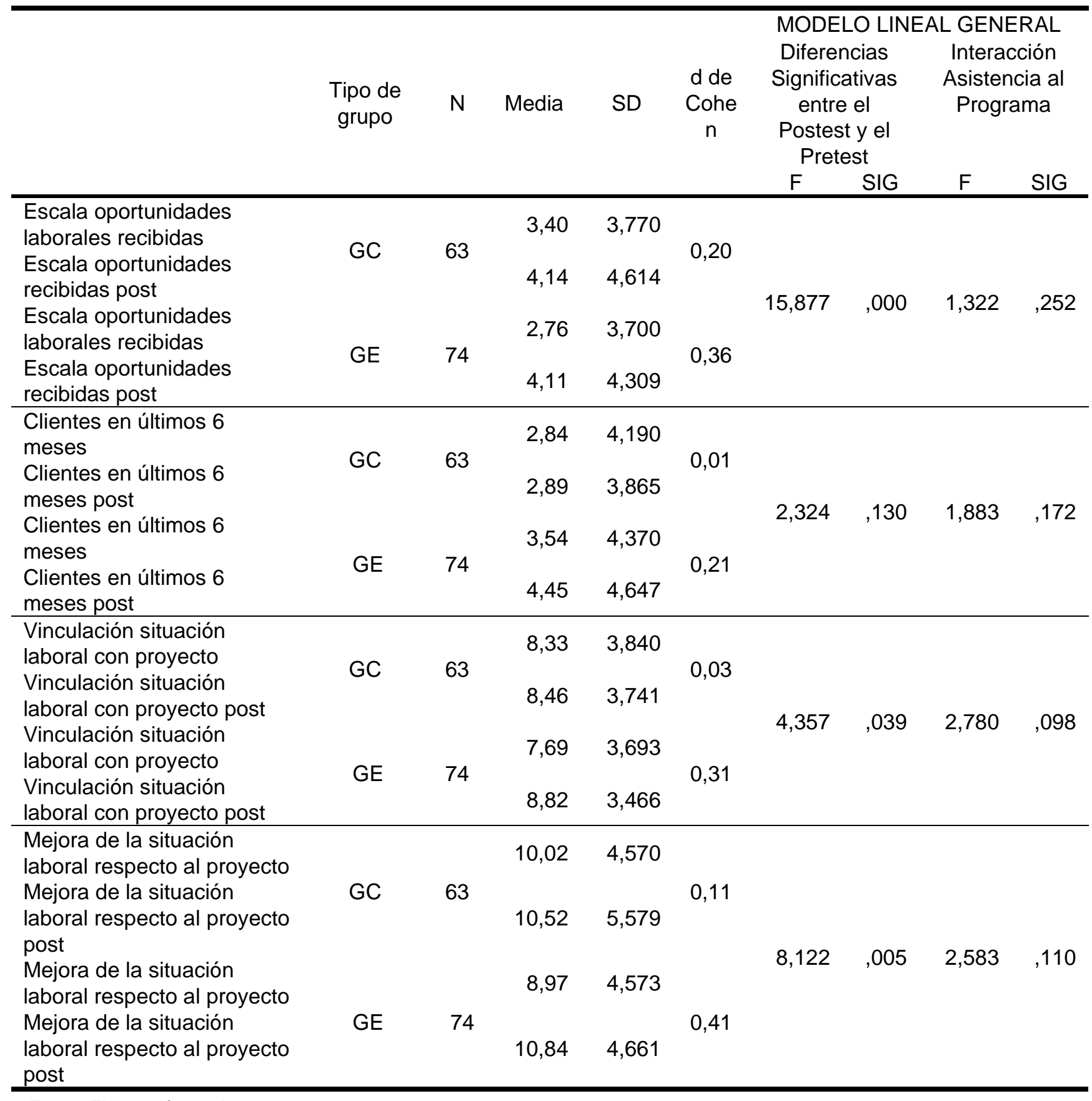

Fuente: Elaboración propia 


\section{Gráfico 1}

Efectos del programa en el GE y el GC

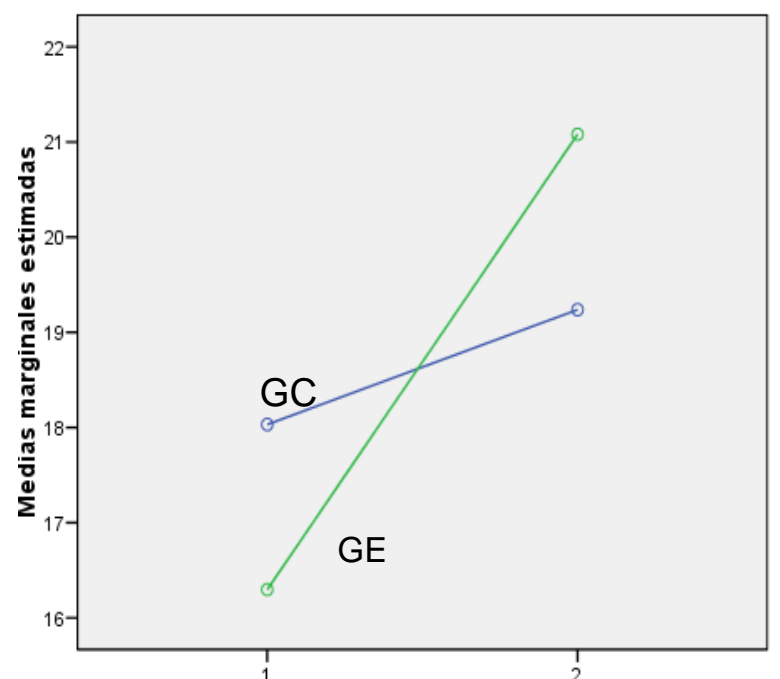

Efecto del programa entre el pretest y el postest en el GE y el GC.

Escala Proyecto Profesional y de la Marca Personal

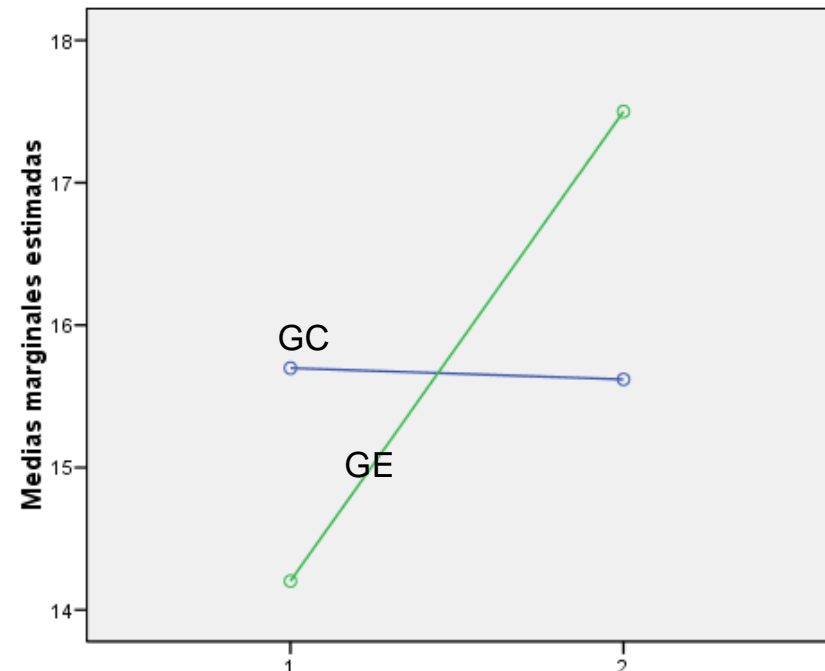

Efecto del programa entre el pretest y el postest en el GE y el GC.

Escala Satisfacción Laboral

Fuente: Elaboración propia

En la figura 2 se presenta el análisis SEM (modelo de ecuaciones estructurales) poniendo como variable independiente la asistencia al programa. Como variables endógenas se han puesto las diferencias de cada una de las variables del estudio (la mejora habida en cada escala); se ha restado la puntuación del pretest al postest.

Se representan las relaciones de efecto por haber asistido al programa, sólo con las variables en donde ha habido diferencias significativas entre el pretest y el postest.

El modelo parece que se ajusta bien a los datos estadísticos resultantes: Chi-square $=21,122$, degrees of freedom = 17 y una probabilidad no significativa de, 273 , por lo que hay un buen ajuste. Dato que es afín con el CMIN $(1,173)$, CFI $(, 978)$ y RMSEA $(, 036)$. 


\section{Figura 2}

Modelo Estructural SEM. Efectos del programa en el grado de mejora de las variables del estudio (postestpretest). Datos de los participantes GE y GC

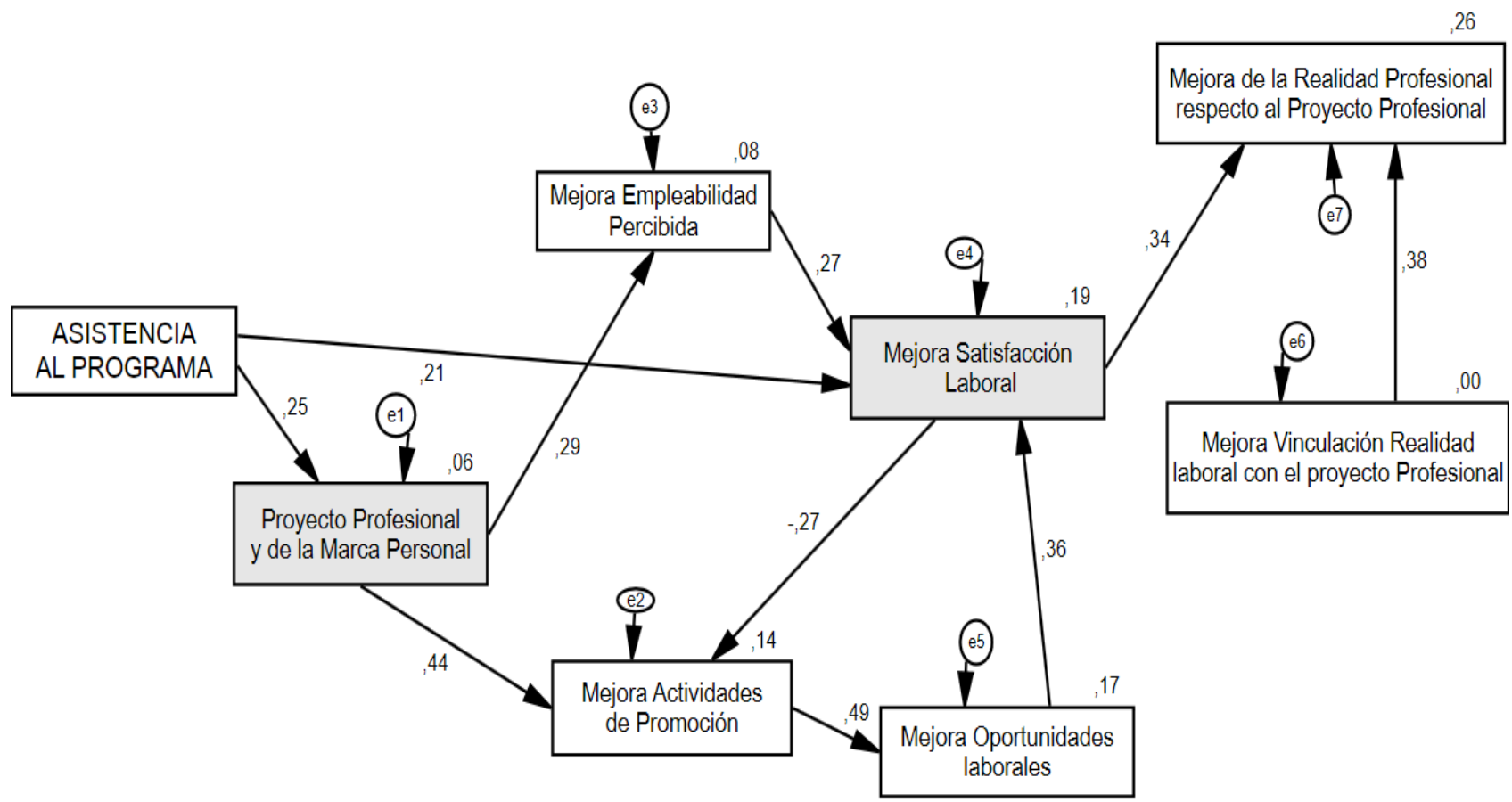

Fuente: Elaboración propia

Analizando la figura, se puede afirmar que el programa ha tenido un efecto directo significativo en las escalas: desarrollo del proyecto profesional y de la marca personal $(B=, 25)$ y en satisfacción laboral $(B=, 21)$. También hay efectos indirectos en: empleabilidad percibida, actividades de promoción, oportunidades recibidas y en la realidad laboral respecto al proyecto profesional.

Revisando el modelo, se podría afirmar que las personas que han asistido al programa han desarrollado más su proyecto profesional y de marca personal, y este ha influido directamente en su satisfacción profesional. El desarrollar el proyecto ha tenido un efecto positivo tanto en su percepción de empleabilidad $(B=, 29)$, como en el incremento de actividades de promoción $(B=, 44)$.

Cuantas más actividades de promoción se realizan, mayores oportunidades laborales se reciben $(B=, 49)$, las cuales permiten sentirse más satisfechos a los participantes $(B=, 36)$. Un resultado inesperado es el efecto negativo de la satisfacción laboral reduciendo las actividades de promoción $(B=-, 27)$. Pudiera ser que las personas que están satisfechas, se promocionan menos por falta de tiempo o porque no lo necesitan.

La mejora de la situación laboral con respecto al proyecto es explicada directamente por la satisfacción laboral $(\beta=, 34)$, y el grado de vinculación del proyecto con la situación laboral (que no es explicada de forma significativa por ninguna variable del estudio). E indirectamente por el resto de variables del estudio. 


\section{Conclusiones}

Los datos obtenidos invitan a afirmar que el programa ha sido eficaz, pues los participantes del GE han mejorado su proyecto profesional y de marca personal; y efectivo, al hallarse efectos al menos 6 meses más tarde de finalizar la parte presencial, con una mejora significativa con respecto al GC.

Los resultados de la investigación son coherentes con los enfoques de gestión personal de la carrera (Greenhaus et al., 2010; Pinto, 2010; Taveira y Rodríguez, 2010), y con los estudios de Llanes et al. (2017), que defienden el incluir estas competencias en los planes de estudio para mejorar la empleabilidad de las personas en formación.

Así mismo, al igual que en la perspectiva de orientación profesional vinculada al desarrollo del proyecto profesional y de vida (Romero, 2004), nuestro profesional tiene un protagonismo esencial en la planificación de su vida laboral y en todas las conductas que forman parte de su plan de acción para conseguir sus metas. El programa permite desarrollar ciertas soft skills muy importantes en la actualidad, como el autoconocimiento, la planificación vital, o la comunicación, obteniendo resultados congruentes con los estudios desarrollados por Rodríguez Martínez et al. (2019) con universitarios egresados. Estas investigaciones enseñan que los programas de orientación mediante el desarrollo de competencias transversales, pueden ser eficaces, aumentando la empleabilidad. También demuestran la importancia de que la orientación profesional impulse el emprendimiento (Palomares et al., 2019). De acuerdo con Rodríguez et al. (2020), se procura que los individuos identifiquen sus fortalezas, estimulen el desarrollo del proyecto profesional, el sentimiento de autoeficacia a la hora de buscar empleo y, finalmente, la mejora de su satisfacción laboral.

Conforme a los resultados de Batistic y Tymon (2017) y de Vanhercke et al. (2014) la empleabilidad percibida ha tenido efectos en variables de empleabilidad. Así mismo, es razonable pensar que el tener un proyecto profesional y de marca personal, aumente dicha empleabilidad percibida, al visualizar el cómo conseguir tus fines laborales. A tenor de los resultados de nuestra investigación, hay indicios de que sí pudiera ser así.

Uno de los valores del estudio es que las variables dependientes son los resultados de la empleabilidad, en comparación con otros programas que, aún siendo muy eficaces y necesarios, las variables consideradas son mediadoras, como por ejemplo el autoconocimiento (Fortuño, 2015) o la adaptabilidad profesional (Santilli et al., 2018); o la satisfacción de los participantes con el programa (Martínez-Pellicera et al., 2014).

Hay muchos autores que hablan de las virtudes de la marca (Arqués, 2019; Deckers y Kyle, 2012; Ferrazzi y Raz, 2020; Pérez Ortega, 2017; Schawbel, 2009; Villaseca, 2017), y otros que venden exactamente lo contrario (Cederberg, 2017; Gershon, 2016; Conley, 2009, Zarkada, 2012). Con esta investigación hemos encontrado evidencias experimentales para conocer lo que realmente hay de cierto en el modelo de branding personal. Los resultados son afines a otros, como los de Gorbatov et al. (2019), donde parece confirmarse que la marca personal conduce a una mayor satisfacción profesional, totalmente mediada por la empleabilidad percibida. Todos estos hallazgos nos permiten concluir que la marca personal es una herramienta fundamental en la actualidad para mejorar profesionalmente.

Tras los resultados, es necesario realizar una serie de preguntas, observaciones y propuestas de investigación futuras. La primera cuestión es por qué no hay ningún efecto significativo, ni en el sentimiento de eficacia profesional, ni en el de reputación profesional percibida. Una explicación puede ser se trata de constructos más estables. Resulta razonable pensar que 6 meses son muy escasos para que alguien pueda sentirse más competente o más importante y popular en su ámbito profesional. Esta cuestión se configura como una línea de trabajo futura. 
Eso sí, hay que tener en cuenta que los estudios cuasiexperimentales, propios de estos diseños de investigación, no permiten el control de todas variables extrañas, por lo que hay que ser prudentes con las conclusiones.

Otras observaciones son la no asignación aleatoria al GE y al GC, y las limitaciones muestrales: los resultados se aplican para un colectivo mayoritariamente femenino, del ámbito de la psicología y la educación. Ello no permite generalizar los datos a otros grupos profesionales. Así, podríamos preguntarnos si este programa podría ser eficaz con perfiles tecnológicos; o, por ejemplo, con profesionales no universitarios. $Y$ hay que ser cautos por el efecto confundido: consideramos de vital importancia el rol y experiencia del profesor que imparta este programa, o el propio efecto de difusión del tratamiento a algunos participantes del grupo control, los cuales manifestaron que a la hora de responder el cuestionario pretest, ya aprendieron cosas para su manera de promocionarse. En este sentido el propio cuestionario podría ser una variable independiente.

También hubiera sido positivo tener sólo una aplicación del programa para todos los participantes experimentales, pero su número elevado, y las recomendaciones metodológicas y pedagógicas obligaban a organizar varios grupos y momentos en los que aplicar el programa.

Por último, otra de las limitaciones de la investigación tiene que ver con el carácter multidimensional de la empleabilidad (Williams et al., 2016), lo que no permite realizar comparativas con otras investigaciones similares por la heterogeneidad de criterios tomados en cuenta (Calvo, 2014).

Dado que las conclusiones de este estudio no pueden generalizarse, sí podrían considerarse futuros estudios que confirmen o enriquezcan estos resultados. Hay una necesidad de comprender un modelo para entender la empleabilidad en el que se puedan identificar realmente los efectos del sentimiento de autoeficacia profesional, la actitud ante el mercado laboral, la empleabilidad percibida y el tener un proyecto profesional y de marca personal, que sea más integrador que el de Vanhercke et al. (2014). O la elaboración y validación de un cuestionario para evaluar el grado de desarrollo del proyecto profesional y de la marca personal. Una herramienta que permita diagnosticar a los participantes e identificar los puntos débiles y fuertes del desarrollo de su proyecto. La construida para este estudio puede ser un comienzo. Otra línea de trabajo que se abre es la de estudios longitudinales a largo plazo con personas que han seguido este tipo de programas.

El cambio ya se ha iniciado. Toca saber si la evolución de la temática acabará con el establecimiento de un modelo de trabajo que suponga un antes y un después en los procesos de orientación profesional. Esta investigación tiene, según nuestro parecer, unas implicaciones prácticas más que significativas. Tiene una relevancia mayúscula por lo que está en juego: la felicidad de las personas, que se merecen laborar en lo que desean, en esta vida singular y única. No sólo tenemos la obligación de elegir qué ser, sino también el tipo de oportunidades que queremos recibir. Se buscaba encontrar una evidencia simbólica de la importancia e influencia del proyecto profesional y la marca personal en la empleabilidad y de la necesidad de seguir trabajando por la construcción de un modelo teórico para comprender los procesos que cualquier persona experimenta en su vida laboral.

\section{Referencias bibliográficas}

Álvarez, M. y Sánchez, M.F. (2017). Concepto, evolución y enfoques clásicos de la orientación profesional. En M.F. Sánchez García (Ed.). Orientación para el desarrollo profesional (pp. 17-64). UNED. 
Arqués, N. (2019). Y tú, ¿qué marca eres? 20 claves para gestionar tu reputación personal. Alienta. Bandura, A. (1977). Social learning theory. Prentice-Hall.

Batistic, S. y Tymon, A. (2017). Networking behaviour, graduate employability: a social capital perspective. Education and training, 59(4), 374-388. https://doi.org/10.1108/ ET-06-2016$\underline{0100}$

Bell, R. (2016). Unpacking the link between entrepreneurialism and employability. An assessment of the relationship between entrepreneurial attitudes and likelihood of graduate employment in a professional field. Education And Training, 58(1), 2-17. https://doi.org/10.1108/ ET-09$\underline{2014-0115}$

Bermúdez López, E., Pérez Navío, E. y Campos Barrionuevo, B. (2020). Orientación Profesional. Nexo entre Competencias Clave y Competencias Laborales. Revista Reflexión e Investigación Educacional, 2(2), 31-52. http://revistas.ubiobio.cl/index.php/REINED/article/view/4120

Calvo, E. (2014). Análisis de la empleabilidad como indicador de la calidad universitaria. En G. Alberti y J.L. Villena. Desafíos y oportunidades de la empleabilidad de los egresados universitarios en el contexto internacional. McGraw-Hill España.

Carroll, A., Houghton, S., Hattie, J., y Durkin, K. (1999). Adolescent reputation enhancement: Differentiating delinquent, nondelinquent and at-risk youths. Journal of Child Psychology and Psychiatry, 40(4), 593-606. https://pubmed.ncbi.nlm.nih.gov/10357165/

Cederberg, C.D. (2017). Personal branding for psychologists: Ethically navigating an emerging vocational trend. Professional Psychology: Research and Practice, 48(3), 183-190. https://doi.org/10.1037/pro0000129

Climent-Rodríguez, J.A. y Navarro-Abal, Y. (2016). Nuevos retos en orientación laboral: de itinerarios personales de inserción a la construcción de marcas profesionales. Revista Española de Orientación y Psicopedagogía, 27(2), 126-133. https://doi.org/10.5944/reop.vol.27.num.2.2016.17148

Conley, L. (2009). OBD: Obsessive Branding Disorder: The Illusion of Business and the Business of Illusion. PublicAffairs

Deckers, E. y Kyle, L. (2012). Branding Personal. Como usar redes sociales para promocionarte. Anaya.

Echeverría, B. (18 de octubre de 2010). Cuestión de saber y sabor. Educaweb. https://www.educaweb.com/noticia/2010/10/18/articulo-competencias-basicas-4418/

Fernández-Salinero, C., y García Álvarez, J. (2020). La inserción laboral de graduados y graduadas a través de los contactos personales. Una propuesta desde la gestión del conocimiento. Teoría de la Educación, 32(1), 163-189. http://dx.doi.org/10.14201/teri.20196

Ferrazzi, K. y Raz, T. (2020). Nunca comas solo. Profit.

Figuera, P. (1996). La inserción del universitario en el mercado de trabajo. EUB.

Fortuño, N. (2015). Diseño, implementación y evaluación de un programa de orientación profesional dirigido a jóvenes que buscan ubicarse en el mercado laboral [Tesis doctoral, Universidad de Zaragoza].

Fugate, M., y Kinicki, A.J. (2008). "A dispositional approach to employability: development of a measure and test of implications for employee reactions to organizational change", Journal 

of Occupational and https://doi.org/10.1348/096317907X241579
organizational
Psychology, 81
(3), 503-527.

Gander, M. (2014). Managing your personal brand, Perspectives. Policy and Practice in Higher Education, 18(3), 99-102. https://doi.org/10.1080/13603108.2014.913538

Gershon, I. (2017). Down and Out in the New Economy: How People Find (or Don't Find) Work Today. University of Chicago Press

Gorbatov, S., Khapova, S.N., y Lysova, E.I. (2019). Get Noticed to Get Ahead: The Impact of Personal Branding on Career Success. Frontiers in psychology, 10, 2662. https://doi.org/10.3389/fpsyg.2019.02662

Greenhaus, J.H., Callanan, G.A. y Godshalk, V.M. (2010). Career management (4ª ed.). Sage Publications.

Hodzic, S., Ripoll, P., Lira, E., y Zenasni, F. (2015) Can intervention in emotional competences increase employability prospects of unemployed adults? Journal Of Vocational Behavior. 88, 28 - 37. https://doi.org/10.1016/j.jvb.2015.02.007

INE (2020). Encuesta de Población Activa (EPA). Segudo trimestre 2020. Instituto Nacional de Estadística.

Izquierdo, T. y Farias, A.J. (2018). Empleabilidad y expectativa de logro en la inserción laboral de los estudiantes universitarios. Revista Española de Orientación y Psicopedagogía. 29(2), 2940. https://doi.org/10.5944/reop.vol.29.num.2.2018.23151

Juárez, J. y Marqués, L. (2019). Aspectos de la competencia digital para la empleabilidad. Revista Española de Orientación y Psicopedagogía. 30(2), 67-88. https://doi.org/10.5944/reop.vol.30.num.2.2019.25339

Krumboltz, J.D., Foley, P. y Cotter, E. (2013). Applying the happenstance learning theory to involuntary career transitions. The Career Development Quarterly, 61,15-25. https://doi.org/10.1002/j.2161-0045.2013.00032.x

Lazarus, R.S. y Folkman, S. (1986). Estrés y procesos cognitivos. Martínez Roca.

Lee Hecht Harrison (29 de marzo de 2017). El mercado laboral oculto: 3 de cada 4 ofertas de empleo en España no son visibles. Adecco. https://www.adeccogroup.es/wpcontent/uploads/2017/07/NdP-Mercado-oculto-en-Espan\%CC\%83a El-75-de-las-ofertasde-empleo-no-es-visible.pdf

Llanes, J., Figuera, P. y Torrado, M. (2017). Desarrollo de la empleabilidad y gestión personal de la carrera de graduados en pedagogía. Revista Española de Orientación y Psicopedagogía. 28(2), 46-60. https://doi.org/10.5944/reop.vol.28.num.2.2017.20118

Maquillón J. (2005). La evaluación del diseño de programas mediante el juicio de expertos: propuesta técnica de un instrumento. En $V$ Congreso Internacional Virtual de Educación. Universidad de las Islas Baleares y Cibereduca. 7-27 de febrero de 2005.

Martínez-Pellicera, A., Llamas-Botíab, A. y García-Palmac, M.B. (2014). La orientación profesional en la Universidad como instrumento para la mejora de la empleabilidad. El caso de la Universidad de Murcia. En XIII Congreso Internacional de Teoría de la Educación. Sevilla. 26-28 de noviembre de 2014.

Natalya, E. y Maria, K. (2013). Undergraduates' expectations on their oncoming employability. 6th International conference of education, research, and innovation (ICERI 2013). Proceedings ICERI, pp. 5352-5361. https://library.iated.org/publications/ICERI2017/start/600 
Nickson, D., Warhust, C., Commander, J., Hurrell, S.A. y Cullen, A.M. (2012). Soft skills and employability; Evidence from UK retail. Economic and Industrial Democracy, 33, 65-84. http://dx.doi.org/10.1177/0143831X11427589

Nisha, S.M. y Rajasekaran, V. (2018). Employability Skills: A Review. IUP Journal of Soft Skills, 12(1), 29-37. https://doi.org/10.7179/PSRI 2021.37.02

OEEU (2015). Barómetro de empleabilidad y empleo de los universitarios en España, 2015. Observatorio de Empleabilidad y Empleo Universitarios.

Palomares-Montero, D., Chisvert-Tarazona, M.J. y Suárez-Ortega, M. (2019). Formación y orientación para el emprendimiento. Lo que dicen la bibliometría y los emprendedores noveles. Revista Española de Orientación y Psicopedagogía. 30(1), 131-149. https://doi.org/10.5944/reop.vol.30.num.1.2019.25198

Pérez Ortega, A. (2015). Marca Personal. Como convertirse en la opción preferente. ESIC.

Pérez Ortega, A. (2017). Marca personal para Dummies. Para Dummies.

Peters, Y. (31 de Agosto de 1997). The Brand Called you. Fastcompany. https://www.fastcompany.com/28905/brand-called-you

Pinto, J. C., Taveira, M. y Ordónez, J.L. (2016). Cómo orientar la gestión de la carrera profesional. Editorial UOC.

Randstad (16 de mayo de 2018). Ocho de cada diez trabajadores menores de 25 años están satisfechos con su empleo. Randstad. https://www.randstad.es/nosotros/sala-prensa/ochode-cada-diez-trabajadores-menores-de-25-anos-estan-satisfechos-con-su-empleo/

Rodríguez Martínez, A., Cortés, A. y Val, S. (2019). Análisis de la mejora del nivel de empleabilidad de los universitarios mediante la mejora de competencias transversales y habilidades. Revista Española de Orientación y Psicopedagogía, 30(3), 102-119. https://doi.org/10.5944/reop.vol.30.num.3.2019.26275

Romero, S. (2004). Aprender a construir proyectos profesionales y vitales. Revista Española de $\begin{array}{lllll}\text { Orientación } & y & \text { Psicopedagogía, } & 15 & \text { (2), }\end{array}$ https://doi.org/10.5944/reop.vol.15.num.2.2004.11637

Romero, V., Pérez, E., Vidal, S. y Juez, M. (2004). Inserción Ocupacional. Altamar.

Rodríguez Esquivel, N.C., y Gallardo Córdova, K.E. (2020). El bienestar y la orientación educativa enfocados en las nuevas generaciones. Revista Española de Orientación y Psicopedagogía, 31(2), 7-18. https://doi.org/10.5944/reop.vol.31.num.2.2020.27982

Sáez, F. y Torres, C. (2007). Empleabilidad. Fundipe. Documento técnico síntesis del encuentro sobre Empleabilidad. http://www.fundipe.es/archives/FOLLEMPL seguro.pdf

Santilli, S., Nota, L. y Hartung P.J. (2019). Efficacy of a group career construction intervention with early adolescent youth. Journal of Vocational Behavior, 111, 49-58. https://doi.org/10.1016/i.jvb.2018.06.007

Savickas, M.L. (2012). Life design: A paradigm for Career intervention in the 21 Century. Journal of Counseling and Development, 90, 13-19. https://doi.org/10.1111/j.1556-6676.2012.00002.x

Scharr, S., y Bartlett, B. (2014). Improving employability and outcomes through workplace learning: case studies of youth in social enterprises. Iceri2014: 7th international conference of education, research and innovation. Iceri Proceedings, pp.3773-3778

Schawbel, D. (2009). Me 2.0: Build a powerful brand to achieve career success. Kaplan Publishing. 
Sobrado, L.M. y Ceinos, C. (2017). Modelos emergentes en orientación profesional. En M.F. Sánchez García (Ed.). Orientación para el desarrollo profesional (pp: 65-100). UNED.

Sobrado, L.M. y Cortés, A. (2009). Orientación profesional. Nuevos escenarios y perspectivas. Biblioteca nueva.

Stumpf, S.A., Colarelli S., y Hartman, K. (1983). Development of the Career Exploration Survey (CES). Journal of Vocational Behavior, 22, 191-226. https://doi.org/10.1016/00018791(83)90028-3

Suárez, B. (2014). La universidad española ante la empleabilidad de sus graduados: estrategias para su mejora. Revista Española de Orientación y Psicopedagogía, 25(2), 90-110. https://doi.org/10.5944/reop.vol.25.num.2.2014.13522

Sun, S., Song, Z. y Lim, V.K. (2013). Dynamics of the job search process: developing and testing a mediated moderation model. Journal of Applied Psychology, 98, 771-784. https://doi.org/10.1037/a0033606

Van der Heijde, C.M. y Van der Heijden, B.I. (2006). A competence-based and multidimensional operationalization and measurement of employability. Human Resource Management, 45, 449-476. https://doi.org/10.1108 / S1877-636120140000014009

Vanhercke, D., Cuyper, N. y De Witte, H. (2016). Perceived employability and well-being: An overview. Psihologia Resurselor Umane, 14, 8-18. https://psycnet.apa.org/record/201628283-002

Vanhercke, D., De Cuyper, N., Peeters, E y De Witte, H. (2014). Defining perceived employability: A psychological approach. Personnel Review, 43(4), 592-605. https://doi.org/10.1108/PR-07$\underline{2012-0110}$

Villaseca, D. (2017). Desarrolla tu talento digital: Cómo acelerar tu carrera y reforzar tu marca personal. ESIC Editorial.

Williams, S., Dodd, L.J., Steele C. y Randall R. (2016). A systematic review of current understandings of employability, Journal of education and work, 29(8), 877-901. https://doi.org/10.1080/13639080.2015.1102210

Zarkada, A. (2012). Concepts and constructs for personal branding: An exploratory literature review approach. http://dx.doi.org/10.2139/ssrn.1994522

Fecha de entrada: 4 marzo 2020

Fecha de revisión: 23 septiembre 2020

Fecha de aceptación: 23 octubre 2020 